\title{
Untersuchung sprachlicher Praktiken in Familien und im Kindergarten: \\ Methodologische und theoretische Grundlagen
}

\section{Dieter Isler und Sibylle Künzli}

"Indem die Schule es unterlässt, durch eine methodische Unterweisung allen das zu vermitteln, was einige ihrem familialen Milieu verdanken, sanktioniert sie die Ungleichheit, die alleine sie verringern könnte.» (Bourdieu, 2001, S. 48)

In der Schweiz besteht schon beim Schuleintritt ein starker Zusammenhang zwischen der sozialen Herkunft der Kinder und ihren sprachlichen Leistungen. Über familiäre Bildungserfahrungen und sprachliche Förderung in vorschulischen Einrichtungen ist aber noch sehr wenig bekannt. Der Beitrag befasst sich deshalb mit methodischen und theoretischen Grundlagen für die Erforschung sprachlicher Praktiken. Im ersten Teil werden methodologische Prämissen und Implikationen behandelt. Im zweiten Teil stellen wir unser theoretisches Verständnis von Sprache als sozialer Praxis dar und veranschaulichen es anhand von exemplarischen Daten aus dem Projekt "Lernwelten - literacies». Abschliessend werden Perspektiven für die sprachdidaktische Unterrichtsforschung und die Professionalisierung von Lehrpersonen skizziert.

\section{Ausgangs lage}

Wie die Resultate der PISA-Studie 2009 zeigen, besteht in der Schweiz nach wie vor ein deutlicher Zusammenhang zwischen den Leseleistungen der 15-Jährigen und der sozioökonomischen Lage ihrer Familien (OECD, 2010). Herkunftsbedingte sprachliche Leistungsunterschiede bestehen bereits zu Beginn der ersten Klasse (Moser, 2005) und werden im Verlauf der Schulzeit noch verstärkt (Moser, Keller \& Zimmermann, 2008). Viele Hoffnungen ruhen deshalb auf der frühen Bildung und insbesondere auf der frühen Sprachförderung (OECD, 2006). Massnahmen in diesem Bereich sollen zum Abbau ungleicher Bildungschancen, zur besseren Integration und zur Verbesserung der Schulleistungen von Kindern und Jugendlichen unterschiedlicher Herkunft beitragen. Für die frühe Sprachförderung werden zurzeit viele neue Angebotsformen, Curricula, 
Diagnoseinstrumente und Fördermaterialien entwickelt und implementiert. Es wird jedoch auch vor überzogenen Erwartungen und Aktivismus gewarnt (Grossenbacher, 2007). Denn über Bildung im Früh- und Vorschulbereich im Allgemeinen und Gelingensbedingungen sprachlicher Förderung im Besonderen ist derzeit noch sehr wenig gesichertes Wissen vorhanden (Ehlich, 2005; Fried, 2010; Rossbach, Klugcniok \& Kuger, 2008; Wannack, Sörensen Criblez \& Gilliéron Giroud, 2006).

Im Rahmen des ethnografischen Forschungsprojekts «Lernwelten literacies» haben wir uns deshalb mit Bedingungen und Prozessen des sprachlichen Lernens 5- und 6-jähriger Kinder befasst. Untersucht wurden sprachliche und literale Praktiken in verschiedenen Familien und im Kindergarten. Im Zentrum stand die Passung dieser Praktiken, d.h. die Verwertbarkeit unterschiedlicher familiärer Bildungserfahrungen im Feld des Kindergartens. Die bisherigen Ergebnisse zeigen, dass das in den Familien erworbene kulturelle Kapital im Kindergarten unterschiedlich anschlussfähig ist, und dass die Lernprozesse der Kinder, aber auch Prozesse ihrer sozialen Positionierung durch den Anschluss an solche Ressourcen unterstützt werden. Umgekehrt können Kinder ihre sprachlichen und literalen Fähigkeiten nicht realisieren, wenn sie durch mangelnde Strukturierung sozialer Situationen oder durch Unvertrautheit mit schulspezifischen Handlungsmustern in ihrer Handlungsfähigkeit eingeschränkt werden. Für eine breitere Darstellung verweisen wir auf die bisherigen Publikationen (Isler \& Künzli, 2008, 2010; Künzli, Isler \& Leemann, 2010).

Die Erforschung sprachlicher Bildungsprozesse 4- bis 6-jähriger Kinder in familiären und schulischen Kontexten ist in verschiedener Hinsicht herausfordernd: Das Feld befindet sich im Schnittbereich der Disziplinen Linguistik, Lernpsychologie und Bildungssoziologie. Dieser Umstand erschwert sowohl die theoretische Modellierung als auch die empirische Untersuchung der Gegenstände. Weil situierte sprachliche Bildungsprozesse zudem von einer Vielzahl von Bedingungen dynamisch beeinflusst werden, und weil darüber bisher sehr wenig gesichertes Wissen vorliegt, sind vorerst offene, verstehende Zugänge notwendig, die der Komplexität der untersuchten Phänomene gerecht werden. Die dazu geeigneten Methoden der qualitativen Sozialforschung sind aber in der sprachdidaktischen Forschung noch wenig verbreitet.

In diesem Beitrag liegt der Fokus deshalb auf der Darstellung methodischer und theoretischer Grundlagen für die Untersuchung sprachlicher Bildungsprozesse in Familien und im Kindergarten. Im zweiten Kapitel geht es um die methodologischen und methodischen Prämissen und Implikationen, die ein dem Gegenstand angemessenes Erforschen erst ermöglichen. Im dritten Kapitel werden theoretische Ansätze dargestellt, die für unser Verständnis von Sprache und sprachlicher Bildung konstituierend sind. Sie werden anhand exemplarischer Beobachtungssequenzen aus dem Projekt «Lernwelten - literacies» veranschaulicht. ${ }^{1}$ Im letzten Kapitel werden Perspektiven für die sprachdidaktische Forschung und die Professionalisierung von Kindergarten-Lehrpersonen skizziert. 


\section{Methodologische und methodische Grundlagen}

\section{Grundverständnis verstehend-rekonstruktiver Sozial- forschung}

Was wird unter verstehend-rekonstruktiver Forschung verstanden? Alle nichtstandardisierten Verfahren der empirischen Forschung gehen von der gemeinsamen Basis der Konstruktion von sozialer Wirklichkeit aus (Flick, 2002). Forschende versuchen, die Konstruktionen der Wirklichkeit, welche die Akteure in ihrem Handeln vollziehen, im Forschungsprozess ähnlich einem mimetischen Prozess zu rekonstruieren. "Mimetische Prozesse sind Prozesse der Nachahmung und des ähnlich Werdens», sie sind jedoch "keine blossen Wiederholungen, sondern Prozesse des (eigenständigen) Nachschaffens»(Wulf, 2006, S. 118). Mimetische Prozesse spielen auch im gesellschaftlichen Handeln von Individuen eine wichtige Rolle, denn darin wird soziales Handeln gelernt (Gebauer \& Wulf, 1998). Solche Lernprozesse laufen für die Handelnden jenseits des Bewusstseins ab und können nicht intentional gesteuert werden. Im alltäglichen, lebenspraktischen und routinierten Handeln geschehen sie wie selbstverständlich nebenbei. Polanyi (1985) spricht vom «tacit knowledge» und meint damit das implizite Wissen, das die Akteure zwar über die habitualisierte Handlungspraxis zur Verfügung haben, um sozial akzeptiert und regelkonform zu handeln, das sie aber selber nicht erläutern oder gar benennen können. Ein Beispiel: Die Lehrpersonen haben sich im Laufe ihrer Ausbildung explizites Wissen angeeignet, sie beherrschen das Unterrichten, und verfügen über Modelle, um beispielsweise Kinder beim sprachlichen Lernen zu unterstützen. Das implizite Wissen, die Regeln der Interaktionen, um von den Kindern, Eltern, Kolleginnen oder Behördenmitgliedern als Lehrperson sozial akzeptiert zu werden oder ihre lebenspraktischen Orientierungen sind ihnen aber häufig nicht bewusst. Sie können deshalb nicht erläutern, mit welchen Mitteln, Prämissen oder Methoden sie ihre alltägliche Lebenswelt konstruieren. Atheoretisches, durch Praxis aufgebautes Erfahrungswissen steht sozial Handelnden nicht als explizites Wissen zur Verfügung.

Die rekonstruktive Sozialforschung ist darauf ausgerichtet, die impliziten Wissensbestände, die nicht bewussten Regeln des Handelns, die innere Logik von gesellschaftlichen Deutungsmustern zu explizieren, d.h. sie zu verstehen und zu erklären. Eltern oder Kindergärtnerinnen können nicht einfach dazu befragt werden, wie sie im Rahmen der Familie oder des Kindergartens handeln, mit welchen Methoden sie den Alltag konstruieren oder was die innere Logik ihres Umgangs mit den Kindern ist. Die Logik ihres Handelns kann aber mittels verstehender rekonstruierender Forschung erschlossen werden. Rekonstruktive Forschung begreift die sozialen Handlungen der Akteure als Dokumente, an denen sich eine Regelhaftigkeit oder innere Logik ablesen und zeigen lässt. Das Erkenntnispotenzial solcher Forschung liegt darin, «soziale Verhältnisse als Sinnzusammenhänge erfassen zu können und auf diese Weise einen verste- 
henden Nachvollzug sozialen Handelns zu ermöglichen» (Meuser, 2006, S. 142). Dazu ist die Ethnografie als Forschungsstrategie hilfreich. Um ein Forschungsfeld von innen heraus zu explorieren, müssen die Forschenden dem Gegenstand im Feld einerseits so nah und andererseits so naiv, offen und neugierig wie möglich gegenübertreten. Ziel ist es, eine Haltung der Fremdheit gegenüber dem Bekannten und Vertrauten zu finden oder wieder zu entdecken (Hitzler, 2006). Hirschauer und Amann (1997) sprechen in diesem Zusammenhang von der Befremdung der eigenen Kultur» (S. 7-52). Die Ethnografie als Forschungsstrategie erfordert ein Hinterfragen von «alten», «fraglosen», selbstverständlichen Überzeugungen und ein Eintauchen ins Forschungsfeld, ohne es stark zu beeinflussen. Es geht um das Verhältnis von Fremdheit und Vertrautheit im Forschungsprozess bzw. um das Aushandeln von Nähe und Distanz (Flick, 2002) - beides, zu grosse Nähe und zu grosse Distanz zum Forschungsgegenstand, kann das Erkennen der Relevanzstrukturen, die dem sozialen Handeln der Akteure im Feld zugrunde liegen, verunmöglichen. Dazu ist es notwendig, die eigenen Subjektivität zu anerkennen und zu reflektieren, indem zum Beispiel das eigene Vorwissen zum Feld expliziert wird.

Der verstehende Nachvollzug von Konstruktionen der Wirklichkeit erfolgt nach den Prinzipien der Abduktion als dem einzig kenntniserweiternden Schlussverfahren (Reichertz, 2006). Die Abduktion schliesst nach bestimmten Prämissen oder aufgrund von Hintergrundwissen auf die plausibelste Erklärung für ein bestimmtes Phänomen oder Ereignis, stellt also durch geschicktes «informiertes Raten» (Reichertz, 2006, S. 13) neue Hypothesen auf und generiert neue Fragen. Die Erkenntnis ist jedoch immer nur vorläufig und kann nie als restlos gesichertes neues Wissen dargestellt werden, denn es kann immer sein, dass der Einzelfall, der die Erkenntnis wieder in Frage stellt oder nochmals verändert, bisher noch nicht in der Untersuchungseinheit enthalten ist. Der Erkenntnisprozess kann deshalb nicht endgültig abgeschlossen werden. Ein abduktives Vorgehen impliziert die Erwartungshaltung, «alte Überzeugungen aufzugeben und neue zu suchen» (Reichertz, 2006, S. 13). Forschende sollen für neue Entdeckungen offen sein. Diese Art des Schliessens kann als kreativ bezeichnet werden, denn sie «ist nicht zwingend, eher sehr waghalsig» (Reichertz, 2006, S. 13). Forschen gleicht dabei der kriminalistischen Tätigkeit, dem Führen eines Indizienprozesses, bei dem die Forschenden möglichst viele «Verdachtsmomente» sammeln, um die plausibelste Lesart oder Erklärung zu finden und zu belegen.

Rekonstruktive Sozialforschung setzt am Einzelfall an (Flick, 2002). Im Rahmen der Fallrekonstruktion versucht sie, ihn in seiner ganze Fülle zu dokumentieren und möglichst umfassend zu durchdringen. Dabei geht es nicht nur darum, den Fall im Sinne einer «dichten Beschreibung» (Geertz, 2003) detailgenau (in allen Einzelheiten, aus allen möglichen Perspektiven) zu beschreiben. Wesentlich ist es, «das Ganze auf den Punkt einer verstehenden Deutung oder eines erklärenden Zusammenhangs» (Bude, 2006, S. 60) zu bringen. Dabei spielt die fallvergleichende Analyse eine wichtige Rolle: Die 
Suche nach Gemeinsamkeiten, Ähnlichkeiten und Differenzen ermöglicht es, das Typische oder die innere Logik eines Falles herauszuarbeiten.

\section{Forschungsperspektiven und Verfahren}

Welche konkreten Erhebungs- und Analyseverfahren eignen sich, um aus diesem Forschungsverständnis heraus frühe sprachliche Bildungsprozesse zu untersuchen? Nach Knoblauch (2008) ist bei der Auswahl der adäquaten Methoden folgender Umstand zentral: «Die Methode ist eine Funktion der Fragestellung ist eine Funktion der Daten ist eine Funktion der Methode.»(S. 4). Die Fragestellung und somit das Erkenntnisinteresse sind massgeblich dafür, welche Auswertungs- und Erhebungsverfahren in Frage kommen und welche Daten wir generieren können. D.h. alles hängt zirkulär zusammen und voneinander $\mathrm{ab}$, die Auswahl der adäquaten Methoden ist nicht beliebig, und sie soll im Laufe des Forschungsprozesses auch im Auge behalten werden. Dabei lassen sich drei theoretische Perspektiven mit je spezifischen Verfahren unterscheiden (Flick, 2002, S. 33-52): Sie sind gerichtet auf 1. die subjektiven Sichtweisen der Akteur/innen, 2. die Herstellung sozialer Wirklichkeit und 3. tieferliegende gesellschaftliche Strukturen. Diese Perspektiven unterscheiden sich in ihrem wissenschaftstheoretischen Hintergrund und Gegenstandsverständnis, aber auch in ihren methodischen Foki, Ansatzpunkten der Analyse und Verallgemeinerungszielen.

Bei der Rekonstruktion von subjektiven Sichtweisen ist die Tradition des symbolischen Interaktionismus als wissenschaftstheoretischer Hintergrund zentral. Die Perspektive fokussiert die subjektiven Bedeutungen und Sinnzuschreibungen, die Individuen mit Handlungen, Erfahrungen und Phänomenen verbinden. Das Ziel solcher Verfahrensweisen ist der Nachvollzug subjektiv gemeinten Sinns. Die Forschung setzt deshalb bei den Subjekten an und versucht, die unterschiedlichen Sichtweisen zu explizieren, «in denen Subjekte Gegenstände, Ereignisse, Erfahrungen etc. mit Bedeutung versehen» (Flick, 2002, S. 35). Dabei handelt es sich um bewusste Vorgänge. Es geht um das Erforschen von Alltagswissen, subjektiven Theorien sowie von subjektiven Repräsentationen von sozialen Phänomenen und damit um konkrete Inhalte. Die «Grounded Theory» (Strauss \& Corbin, 1996) bietet Verfahren an, die für die Rekonstruktion von Alltagswissen und subjektive Sichtweisen besonders geeignet sind. Aus dieser Perspektive fragen Forschende beispielsweise nach den subjektiven Theorien von Kindergarten-Lehrpersonen zu sprachlicher Bildung, um eine datenbegründete Theorie zu entwickeln.

Die zweite Perspektive, die auf dem wissenschaftstheoretischen Hintergrund der Ethnomethodologie basiert, ist an den Routinen des Alltagshandelns und an den Herstellungsprozessen von sozialer Wirklichkeit interessiert. Die Ethnomethodologie befasst sich mit der für die Soziologie zentralen Frage: Wie ist überhaupt soziale Ordnung und damit soziale Wirklichkeit möglich? Wie stellen wir in unserem alläglichen Tun soziale Wirklichkeit her? Sie geht dabei von der 
Vollzugswirklichkeit als Prämisse aus, d.h. die Wirklichkeit existiert nur in den Handlungen der Gesellschaftsmitglieder. Wirklichkeit wird durch die Beteiligten an Ort und Stelle (lokal), aus der Situation und dem Verlauf der Handlung heraus (endogen) und mittels der gegebenen Ausdrucks- und Wahrnehmungsmöglichkeiten (audiovisuell) erzeugt (Garfinkel, 1967). Das Hauptziel dieser Perspektive ist es, das soziale Handeln zu beschreiben und die wirklichkeitsherstellenden Regeln zu rekonstruieren. Aus dieser Perspektive setzt Forschung bei den Interaktionen zwischen sozial Handelnden bzw. bei den Diskursen an und versucht, die Selbstverständlichkeiten zu explizieren, die den Handelnden beim Interagieren oft gar nicht bewusst sind. Das «Wie» der sozialen Handlung, die verwendeten Formen und deren Rolle bei der Herstellung sozialer Wirklichkeit stehen im Zentrum der Analyse. Dazu eignen sich konversations-, diskurs-, und videoanalytische Verfahren, die das Ziel verfolgen, verschiedene Typen von Herstellungsregeln oder von Handlungsformen zu unterscheiden.

Schliesslich die Ausrichtung, die sich auf strukturalistische Positionen bezieht und die von Prozessen des sozialen Unbewussten ausgeht. Die Analyse in dieser Perspektive ist an der Rekonstruktion von gesellschaftlichen Deutungs- und Handlungsmustern interessiert, sie möchte die handlungs- und bedeutungsgenerierenden Tiefenstrukturen (Flick, 2002) rekonstruieren und setzt dabei bei den kulturellen Sinnsystemen oder -strukturen an, «die die Wahrnehmung und Herstellung subjektiver und sozialer Wirklichkeit gleichsam rahmen» (Flick, 2002, S. 43). Dabei wird zwischen einer Oberflächen- und einer Tiefenstruktur unterschieden. Der oberflächliche Informationsgehalt des Erlebens und Handelns ist den beteiligten Subjekten zugänglich, die tieferliegenden kulturellen Sinn- und Bedeutungsschichten werden von der alltäglichen, individuellen Reflexion der Beteiligten nicht erreicht und sollen durch die Analyse rekonstruiert werden. Forschung in dieser Perspektive setzt bei den unbewussten Strukturen an und versucht sie mit dem Ziel des Herausarbeitens einer Fallstruktur oder einer inneren Logik des Falles zu explizieren, um daraus Hinweise auf unterschiedliche Typen zu erfahren. Die verwendeten Analyseverfahren sind u.a. die Objektive Hermeneutik, die Dokumentarische Methode der Interpretation sowie die Typenbildende Habitushermeneutik. Eine Fragestellung aus dieser Perspektive könnte sein: Welche Handlungsstruktur(en) ist / sind im Bildungsverständnis von Kindergärtnerinnen erkennbar?

Wir haben drei grundlegenden Perspektiven skizziert, die alternative Möglichkeiten eröffnen, um die soziale Wirklichkeit forschend zu rekonstruieren. Dabei zeigen sich die Potenziale, aber auch die Grenzen der unterschiedlichen Analyseverfahren. Die Begrenztheit der einzelnen Methoden kann durch Triangulation der Perspektiven ein Stück weit aufgehoben werden. Diese Art der Triangulation hat sich innerhalb der rekonstruktiven Sozialforschung als Anspruch etabliert, um der Komplexität von Forschungsgegenständen gerecht $\mathrm{zu}$ werden und zu einer umfassenderen Erkenntnis zu gelangen. Triangulation 
meint dabei, einen "Forschungsgegenstand von (mindestens) zwei Punkten aus» (Flick, 2008, S. 11) in den Blick zu nehmen. Die Kombination zweier Perspektiven und damit zweier Erhebungs- und Analyseverfahren spielt besonders in ethnografischen Ansätzen eine immer wichtigere Rolle (Flick, 2006, S. 161).

Im Projekt «Lernwelten - literacies» haben wir im Sinne einer ethnographischen Forschungsstrategie die sprachlichen und literalen Praktiken in Familien und im Kindergarten untersucht. Wir haben in den Familien und Kindergärten teilnehmend beobachtet, mit den Akteur/innen Gespräche geführt und am Ende der Erhebungsphasen den Lernstand der Kinder im Lesen erhoben. Aus der ersten Perspektive haben wir die subjektiven Bedeutungen herausgearbeitet, die Kindergärtnerinnen und Eltern mit sprachlicher Bildung verbinden, aus der zweiten konnten wir das Wie des sprachlichen Handelns explizieren und so für Veränderungsprozesse zugänglich machen. Nicht berücksichtigt haben wir die dritte Perspektive, die nach den tieferliegenden Strukturen fragt.

\section{Theoretische Grundlagen}

Für die Sprachdidaktik spielen traditionellerweise Theoriebezüge zu Linguistik, Literaturwissenschaft und Lernpsychologie eine zentrale Rolle: Es geht um die Frage, wie Wissen über Sprache und Literatur sowie sprachliches Können möglichst allen Lernenden möglichst effektiv vermittelt werden. Dabei wird Sprache als Medium des Unterrichts in allen Fächern und als Gegenstand der Sprachfächer konzeptionalisiert (Felder, 2003). Im Zusammenhang mit der aktuellen bildungspolitischen Bedeutung der frühen Sprachförderung steht die Sprachdidaktik heute vor neuen Aufgaben: Erstens rücken neben der Schule auch der Kindergarten ${ }^{2}$, verschiedene weitere pädagogische Berufsfelder (z.B. Krippen oder Spielgruppen) und der familiäre Alltag in ihren Blick. Zweitens erfordern die vielfach belegten Zusammenhänge zwischen sprachlichen Fähigkeiten und sozioökonomischer Herkunft der Lernenden einen stärkeren Einbezug soziologischer Perspektiven.

Im Projekt «Lernwelten - literacies» haben wir uns mit sprachlichen Praktiken in Familien und Kindergärten und ihrer Bedeutung für die Reproduktion bzw. den Abbau von Bildungsungleichheit befasst. Im Folgenden werden einige theoretische Ansätze dargestellt, die sich im Verlauf dieser Arbeiten als besonders bedeutsam erwiesen haben. Ziel ist es, im Hinblick auf eine Sprachdidaktik des Vorschulalters das Potenzial eines stärkeren Einbezugs soziologischer Perspektiven zu verdeutlichen.

\section{Sprachliche Interaktion als soziales Handeln}

In der sprachdidaktischen Lehre und Praxis zeigt sich oft die Vorstellung von Lehrpersonen, es gäbe eine dem sprachlichen Zeichen (als Wort, Äusserung, Text) innewohnende objektive, von den beteiligten Subjekten unabhängige 
Bedeutung. Dabei wird ausgeblendet, dass Verständigung zwingend ein Prozess der Kokonstruktion ist. Sozialwissenschaftliche Interaktionstheorien können hier zur weiteren Klärung beitragen. Im Folgenden werden einige wichtige Stationen der Theorieentwicklung skizziert. Die Grundlage bildet der für Linguist/innen äusserst hilfreiche historische Abriss von Peter Auer (1999). ${ }^{3}$

Ausgangspunkt ist die Einsicht, dass das Gemeinte nicht nur durch das Sprachsystem, sondern immer auch durch den aktuellen Sprachgebrauch bestimmt wird. Beneviste postuliert deshalb eine Theorie der Äusserung (énonciation): Im Akt des Sprechens erschafft die Sprecherin sich selbst, ihr Gegenüber und den Bezug zur Welt; das Subjekt konstituiert sich in der Sprache durch sein Handeln (Auer, 1999). Während Beneviste Interaktion noch stark vom Sprecher ausgehend und auf Sprache fokussiert beschreibt, richtet Grice seine Aufmerksamkeit stärker auf das Verstehen. Die realisierte sprachliche Äusserung ist aus dieser Perspektive nicht Ausgangspunkt oder primäre Quelle der Verständigung, sondern dient im Zusammenspiel mit anderen Quellen wie Situation, Körpersprache und gemeinsamen Wissensbeständen dem übergeordneten Ziel, sich so zu verhalten, dass die Zuhörerin aus dem Gesagten auf das Gemeinte schliessen kann (S. 93ff.). Nach Schütz geht es beim sozialen Austausch darum, den subjektiven Sinn so in äusserlich wahrnehmbares Verhalten umzusetzen, dass er durch andere unter Einbezug konventioneller Wissensbestände rekonstruiert werden kann. Schütz spricht in diesem Zusammenhang vom "objektiven Sinn» einer sozialen Handlung, der weder durch die konventionellen Bedeutungen der sprachlichen Äusserung noch durch die Absichten der Sprecherin bestimmt ist, sondern durch das Gelingen der Verständigung, der Sinn-Ko-Konstruktion im gemeinsamen, schrittweisen Handlungsvollzug (S. 121ff.). Garfinkel beleuchtet mit dem Begriff der Indexikalität die untrennbare Verwobenheit von situativem Kontext und sprachlicher Äusserung. Mit Reflexivität bezeichnet er die Rückbezogenheit einer fortschreitenden Handlung auf vorangehende Handlungsschritte und damit den prozesshaften Charakter der Sinnkonstruktion (S. 130ff.). Dieser selbstorganisierende Aspekt des Handelns wird von Sacks als Sequentialität gefasst und als Konversationsanalyse weiter ausdifferenziert (diese Methode untersucht die Sinn-Relationen aufeinanderfolgender Handlungspaare, Reparaturen wie Elaborieren, Detaillieren und Reformulieren sowie die interaktive Steuerung des Sprecherwechsels) (S. 138ff.). Während Schütz, Garfinkel und Sacks durch Mikroanalysen von Interaktionen die Herstellungsregeln intersubjektiven Sinns untersuchen, befasst sich Luckmann mit grösseren Einheiten des sprachlichen Handelns, den kommunikativen Gattungen. Diese gesellschaftlich (historisch und kulturell) ausgeformten Handlungsmuster bieten den beteiligten Akteur/innen einerseits Orientierung und Entlastung, schränken sie durch ihre Verbindlichkeit aber auch ein. Sie werden durch eine Binnenstruktur (die Konstellation sprachlicher und textueller Merkmale), eine Aussenstruktur (die Anschlussfähigkeit an spezifische 
Milieus und Situationen) und eine Zwischenebene der sequentiellen Ablaufschemata (zur Steuerung von Rollen, Themen, Rederecht u.a.) charakterisiert (S. 177).

\section{Protokol lauszug}

ADRIAN: "Oder ich wär mit mim ... Polizeiautöli duregfahre. @» BORIS: "Gsesch, so ich eso. ... Det hetsch jetzt mich no gseh, so: Zägg." ADRIAN: "Wuuhu!» BORIS: "Ich hett ... uf de Erde, nachhär ...» ADRIAN: «Du meinsch uf Stei?» BORIS: "Ja, uf em Stei. ... Wruumumäähm. Ich wär jetzt zflitze cho. ... Gesch, ich eso: Zägg, wuähmm!» ADRIAN: «Wieso wärsch du nöd here gfahre?» BORIS: "Ich wär ebe so schnäll gsi, da hett i nüme chöne brämse, und da wär-zagg.»ADRIAN: «Jipie. Wuhu!»BORIS: "@Da gsesch so- nei, ich eso: Wääh-iiih!» ADRIAN: "Hol dir doch au na en Legomannsgöggel.» BORIS: "Nei.» ADRIAN: «Wieso nöd?» BORIS: "Ich wär so- ich wär de gsi, wo so: Pkh!» ADRIAN: «Du wärsch's Auto gsi? ...... 's Auto wo chan flü̈̈ge. @»BORIS: «Nei.... Zum Spile hett ich scho eine gha, aber ich wett jetz keine go hole." ADRIAN: «Ok, dänn nimm ich de au nöd. @ ... Dä wär en Tote gsi."

29BTF, 86-94

\section{Kommentar}

Boris (6 Jahre) und sein Freund Adrian (9 Jahre) spielen zuhause mit Spielzeugautos auf dem Fussboden. Die sprachlichen Äusserungen sind eng mit der Spielhandlung verwoben (Manipulieren der Fahrzeuge, Produktion von Geräuschen). Sie dienen der Markierung von Fiktionalität (Konjunktiv), der kontinuierlichen Herstellung eines gemeinsamen Spieltextes (Bedeutung von Objekten klären, Motive von Figuren klären), der Reparatur sowie dem Aushandeln von Rahmenbedingungen des Spiels (Braucht es Spielfiguren oder nicht?) und des sozialen Vertrags (Adrian lenkt ein und integriert seine Lösung in den Spieltext). Das Beispiel veranschaulicht die Aspekte Multimodalität, Sinn-Kokonstruktion, Indexikalität und Sequentialität. Es stellt für Adrian und Boris eine vertraute kommunikative Gattung dar, die ihnen ein schnelles, innovatives Spiel ermöglicht.

Beispiel 1: Interaktive Herstellung eines fiktionalen Spieltexts als soziales Handeln. Transkription als «eye dialect» (Kowal \& O'Connell, 2008) bzw. lautorientierte Umschrift (@steht für: lachen)

\section{Spracherwerb als kulturelles Lernen und sprachliche Handlungs formate}

Als arbiträres Zeichensystem ist die Sprache prinzipiell unbegrenzt modulierbar und deshalb besonders geeignet zur Darstellung beliebiger Inhalte. Als Werkzeug der Kommunikation und der Kognition verbindet sie situiertes soziales Handeln 
(Diskurs) und mentale Strukturen und Prozesse (Schemata und Skripts) (Holland \& Cole, 1995, S. 485). Damit wird Sprache - im Verbund mit anderen, weniger universellen Zeichensystemen - zum Leitmedium der Aneignung kulturell (nicht biologisch) tradierter Fähigkeiten. Erworben und ausdifferenziert werden kulturelle Fähigkeiten im Kontext sozialer Handlungen mit kompetenten Anderen. Wygotski beschreibt diesen Aneignungsprozess als Interiorisation, d.h. als Verinnerlichung zunächst sozial kokonstruierten (und dabei objektivierten) Wissens und Könnens (Wygotski, 1934/1986, S. 354). Nach Tomasello (1999) müssen für sprachliches Lernen vier Bedingungen erfüllt sein: 1. Das Kind versteht andere Personen als intentional handelnde Wesen. 2. Es besteht eine Situation der geteilten Aufmerksamkeit. 3. Das Kind erkennt die kommunikativen Absichten der anderen Person. 4. Es erhält Gelegenheit zum Rollentausch, um sein Verstehen sozial zu verifizieren (S. 107). Von diesen Bedingungen ist nur die erste von der biologischen Entwicklung des Kindes abhängig und wird in der Regel im Alter von ca. 9 Monaten erreicht (Tomasello, 1999, S. 61). Die anderen Bedingungen werden interaktiv hergestellt.

Die interaktive Sinnkonstruktion gelingt besonders gut, wenn Handlungen in wiederkehrenden Situationen relativ ähnlich ablaufen. Bruner bezeichnet solche ritualisierten Handlungen als Formate (Bruner, 1983/2002, S. 115). Formate sind habituell ausgeformte Muster, die typische sprachliche Handlungssituationen (z.B. das gemeinsame Betrachten eines Bilderbuchs oder das Erzählen eines Erlebnisses) strukturieren, als Schemata und Skripts inkorporiert und als Ressourcen in vergleichbaren Situationen genutzt werden. Wenn ein Kind mit einem Handlungsmuster bereits vertraut ist, kann es seine Aufmerksamkeit sehr viel besser auf Variationen einzelner sprachformaler und inhaltlicher Merkmale lenken. Im institutionellen Kontext der (Vor-)Schule spielen schulische Formate als Rituale eine wichtige Rolle: Indem sie regelmässig in ähnlicher Form durchgeführt werden, ermöglichen sie den Auf- und Ausbau geteilter Bedeutungsräume.

\section{Der Kindergarten als ort der ersten schulischen Sozialisation}

Der Kindergarten ist aus soziologischer Sicht nicht nur eine Organisation, sondern auch eine Institution, d.h. eine "Sinneinheit von habitualisierten Formen des Handelns und der sozialen Interaktion, deren Sinn und Rechtfertigung der jeweiligen Kultur entstammen und deren dauernde Beachtung die umgebende Gesellschaft sichert» (Gukenbiehl, 2002, S. 144). Hier zeigt sich eine wichtige Schnittstelle psychologischer und soziologischer Theorien: Bildungsprozesse werden durch Handlungsformate strukturiert, die institutionenspezifisch ausgeformt sind (und ihrerseits Institutionen ausformen). Thévenaz-Christen (2005) charakterisiert die institutionelle Kultur der Schule als «forme scolaire» (S. 67). Diese schulische Form unterscheidet sich von der alltäglichen (oder informellen) Form durch ihren indirekten Weltbezug (die 
schulischen Lerngegenstände sind in der Regel distant oder abstrakt und müssen repräsentiert werden), die spezifische Organisation von Raum (Schulhaus, Klassenzimmer, Arbeitsplatz), Zeit (Schuljahr, Wochenstundenplan, Lektionssequenzen) und Gegenständen (Fächer, Curricula) sowie die rollenbasierte, didaktische Beziehung zwischen Lehrperson und Klasse (als Kollektiv der einzelnen Kinder). Diese Merkmale hat Thévenaz-Christen (2005) in der Vorschule (école enfantine) nur selten beobachtet: Die Lehr-Lernprozesse sind noch weitgehend global, immersiv und auf unmittelbarer Erfahrung begründet (S. 390). Sie schliesst daraus, dass die Vorschule als Transitionsraum von alltäglichen Sozialisations- und schulischen Lernformen geprägt ist (Thévenaz-Christen, 2005, S. 400). Unsere eigenen Untersuchungen haben bestätigt, dass das Neben- und Miteinander alläglicher und schulischer Formen auch für Deutschschweizer Kindergärten konstituierend ist (Isler \& Künzli, 2010). Das Fehlen einer eigenständigen vorschulischen Form darf allerdings nicht als Defizit missverstanden werden: Es ist im Gegenteil sehr wichtig, dass im Kindergarten sowohl alltägliche als auch schulische Formen ihren festen Platz haben, damit alle Kinder viel Gelegenheit erhalten, um sich ausgehend von (oft) vertrauten alltäglichen Praktiken allmählich neue schulische Praktiken anzueignen.

\section{Protokollauszug}

Während die anderen Kinder lärmen, sitzt Dorian im hinteren Teil des Raumes auf einer Matratze und 'liest' ein Buch. Er hat das Bilderbuch auf den Knien, schaut lange konzentriert auf eine Doppelseite, deutet mit dem Finger auf bestimmte Tiere. Nora stellt sich vor Dorian, deutet auf ein Tier und sagt: "Elefant." Dorian klappt das Buch zu, und Nora geht weg. Dann macht er es wieder auf und beginnt von vorne. Sorgfältig fährt er mit dem Finger über die erste Doppelseite. Auf der nächsten Seite angekommen, betrachtet er einen Teil der Seite lange und aufmerksam. Dann blättert er weiter, verweilt aber jeweils ziemlich lange bei einzelnen Bildern.

B1TBU, 339-350

\section{Kommentar}

Dorian schaut sich in der Kindertagesstätte ein Bilderbuch an. Er ist mit dem Handlungsformat «ein Bilderbuch anschauen» vertraut. Er kann Schrift noch nicht dekodieren, nutzt das Bilderbuch aber funktional als bedeutsamen Text, den er in einer zerdehnten Interaktion (der Autor bzw. die Illustratorin sind räumlich, zeitlich und personal distant) monologisch rezipiert. Innerhalb des musterhaften Handlungsformats kann er sich auf den spezifischen Inhalt dieses Buches fokussieren. Er hat dieses Format gewählt und stellt es nach einem Unterbruch wieder her. Das Beispiel veranschaulicht Funktionen von vertrauten Handlungsformaten (Bruner) bzw. kommunikativen Gattungen (Luckmann). Die Binnenstruktur besteht hier in der Text- bzw. Mediensorte "Bilderbuch», die Aussen- 
struktur in der situativen (zeitlichen, räumlichen, personalen, normativen) Passung von Handlung und Milieu (Kindertagesstätte), die Zwischenebene in den Rollen, die zu besetzen (und zurückzuweisen) sind und den Prozeduren des Blätterns, Betrachtens und Berührens.

Beispiel 2: Schulsprachliches (hier: literales) Handlungsformat im Kontext einer Kindertagesstätte

\section{Schulische Sprache - schulsprachliche Fähigkeiten}

Von besonderer Bedeutung und Ausformung ist auch die Sprache der Schule. Als Unterrichtsmedium hat sie im Klassenzimmer oft die Funktion des öffentlichen Sprechens und Zuhörens (Erard und Schneuwly verwenden dafür den Begriff des «oral publique» (2005, S. 73)): Die Lehrperson interagiert im Unterricht häufig mit einem Klassen- oder Gruppenkollektiv, und die Schülerinnen und Schüler äussern sich vor dem Publikum ihrer Mitschüler/innen. Daraus ergeben sich grundlegend andere Bedingungen der Herstellung und Aufrechterhaltung geteilter Aufmerksamkeit und damit auch andere Anforderungen an die sprachliche Ausformung der Interaktionen. Schulisches Sprechen und Zuhören weist deshalb häufig Merkmale konzeptioneller schriftlicher Sprache auf (Koch \& Oesterreicher, 1994): Sie ist typischerweise kontextunabhängiger, monologischer, expliziter, korrekter und reflektierter als konzeptionell mündliche, situierte Alltagssprache (Feilke, 2002). Zudem ist Sprache schon im Kindergarten und während der gesamten Schulzeit nicht nur Medium, sondern auch Unterrichtsgegenstand von zunehmender Abstraktheit und Komplexität.

Bezüglich des Erwerbs schulsprachlicher Fähigkeiten ist davon auszugehen, dass diese formal-öffentliche bzw. konzeptionell schriftliche Sprache nicht durch immersiven Gebrauch in Alltagssituationen erworben wird, sondern durch Herauslösen aus der kommunikativen Praxis, Thematisieren und Einüben ihrer Merkmale im Rahmen einer didaktischen Vermittlung (Erard \& Schneuwly, 2005). Solche schulischen Lernprozesse stehen in engem Zusammenhang mit dem spezifischen Bildungsauftrag der Schule und sind in anderen, stärker sozialpädagogisch ausgerichteten Institutionen weniger verbreitet (Giraudeau \& Florin, 2002; Hudelot \& Froment, 2006). Hingegen ist davon auszugehen, dass sie in schulbildungsorientierten Familien bereits vor dem Eintritt in den Kindergarten und während der gesamten Schulzeit vermittelt und erworben werden (Cairney \& Ashton, 2006; Künzli, Isler \& Leemann, 2010).

\section{Sprachlicher Habitus und schulisches Feld}

Vorgängig wurden die spezifischen Formen der schulischen Sprache und des Kindergartens je separat dargestellt. Bourdieus Theorie von Habitus und Feld modelliert nun deren Zusammenspiel im Hinblick auf die Herstellung und Aufrechterhaltung sozialer Ordnung bzw. gesellschaftlicher Macht. Sprache ist eine wichtige Form des kulturellen Kapitals und als Distinktionsmittel schon in 
den ersten Schuljahren von grösster Bedeutung (Bourdieu, 2001; Käsler, 2005). Im Rahmen alltäglicher Lernprozesse erwerben Kinder bestimmte sprachliche Praktiken mit spezifischen Merkmalen auf allen Ebenen des Sprachsystems und des Sprachgebrauchs. Diese Praktiken oder "ways with words» (Heath, 1983) sind geprägt von der Geschichte, Ethnie und sozialen Position der Familie und eng verwoben mit ihren ökonomischen Bedingungen und Wertorientierungen (Lahire, 1995). Als inkorporierte Schemata sind sie Teil des Habitus und damit nur bedingt rational kontrollierbar. Wenn Kinder sprachlich handeln, greifen sie auf ihre habitualisierten Praktiken zurück und sind deshalb nicht nur als Individuen, sondern immer auch als Mitglieder bestimmter sozialer Gruppen erkennbar (Bernstein, 1988). Ob sie ihr kulturelles Kapital erfolgreich einsetzen können, wird weitgehend durch die Regeln des jeweiligen Feldes bestimmt, in dem sie handeln. Die Schule bewertet gewisse Praktiken höher als andere, und die Vertrautheit mit der "schulischen Form» (s. oben) bzw. das Verfügen über schulspezifische Sprachfähigkeiten dürfte massgeblich zur Bildungsungleichheit von Kindern unterschiedlicher sozialer Herkunft beitragen.

Käsler (2005) spricht von Sprachbarrieren, wenn Schülerinnen und Schülern aufgrund ihrer sprachlichen Varietät der Zugang zu sozialen Räumen oder schulischen Inhalten verwehrt wird und die Schule keine Anstrengungen unternimmt, um die erforderliche Varietät zu erwerben. Sie führt als Beispiele die schlechtere Bewertung vergleichbarer sprachlicher Leistungen und die fehlende Unterstützung von Kindern mit Deutsch als Zweitsprache beim Zugang zu Lerninhalten an. Im Hinblick auf die Sprachförderung in Deutschschweizer Kindergärten und mit Bezug auf die oben referierten Theorien lassen sich unschwer weitere Mechanismen ausmachen: Augenfällig ist die Verwendung des Dialekts als Unterrichtssprache in Klassen mit hohem Anteil an Kindern mit Deutsch als Zweitsprache. Heimtückischer (und langfristig vermutlich gravierender) ist es, wenn schulische Handlungsformate wie Erlebnisse erzählen, Bilderbücher betrachten, Bastelanleitungen ausführen oder Klassengespräche führen im Kindergarten stillschweigend vorausgesetzt und deshalb auch nicht vermittelt werden. Ähnliches gilt für die Förderung von hierarchiehöheren Fähigkeiten zur (zunächst mündlichen, später zunehmend schriftlichen) Rezeption und Produktion komplexer Texte und von Fähigkeiten im Bereich der Sprachreflexion. Diskriminierung kann im schulischen Feld aber auch von Peers ausgehen, die soziale Positionierungen aushandeln und mit Strukturen des Sozialraums Schule experimentieren.

Protokol lauszug

Die drei Mädchen reden beim Malen miteinander. Irene erzählt von einem anderen Mädchen, das nicht so gut zeichnen kann und immer nur "RoboterRoss» zeichnet. Anna fragt: "Findsch das da schön?" Jana und Irene antworten: "Ja." Anna sagt: "Wenn ich tue Rösser zeichne, werded's immer gruusig!» Dann klatscht sie laut auf ihre Stiftschachtel, und Irene macht ihr 
das nach. Danach sagt Anna: "Weisch was? Min Cousin isch scho mal ufträte. Er spilt Schlagzüg." Jana sagt: "Ich bin au scho ufträte, im Stadttheater." Anna entgegnet: "Ja, aber weisch mit em Schlagzüg, mit andere wo Posune und Bass spiled." Jana versucht noch mehrmals, von ihrem Auftritt zu erzäblen, wird aber von Anna dauernd unterbrochen. Anna dominiert das Gespräch. Manchmal flucht sie: "Gopferdammi!", oder sie spielt mit der Sprache: "Yes and Jazz and Ness.» BF28, 58-67

\section{Kommentar}

Jana sitzt mit Anna und Irene im Kindergarten am Maltisch. Anna positioniert sich als Gruppenchefin. Sie holt sich die Bestätigung der beiden anderen und setzt sich dann über deren Urteil hinweg. Als sie von ihrem bühnenerprobten Cousin erzählt, trumpft Jana mit einem eigenen Theaterauftritt auf. Anna verteidigt ihre Position vehement, zunächst durch Argumente, dann durch Verweigerung des Rederechts, Missachtung der Sprachregelung im Kindergarten und Besetzen des sprachlichen Terrains. Jana hat als Einzelkind wenig Erfahrung mit solchen Positionskämpfen, gibt aber nicht so schnell auf (und wird im weiteren Verlauf des Gesprächs noch einen Teilerfolg erringen). Die Sequenz veranschaulicht einen Positionierungskampf unter Peers, bei dem sprachliches Kapital ins Feld geführt und verwertet wird.

Beispiel 3: Aushandeln sozialer Positionen unter Peers im Feld des Kindergartens

\section{Ausblick}

\section{Perspektiven für die sprachdidaktische Forschung}

Um Prozesse der frühen Sprachförderung zu untersuchen, sind angesichts des aktuellen Forschungsstands zunächst verstehend-rekonstruierende Zugänge notwendig, die das komplexe Zusammenspiel von institutionellen Rahmenbedingungen, heterogenen Bildungsressourcen und Lehr-Lernprozessen berücksichtigen. Der Beizug soziologischer Theorien ermöglicht eine breitere disziplinäre Verankerung der sprachdidaktischen Forschung und ein vollständigeres Verständnis der Zusammenhänge zwischen schulsprachlichen Fähigkeiten und Bildungsungleichheit. Sprache ist aus dieser Perspektive nicht nur Medium und Gegenstand des Unterrichts, sondern auch Mittel der Distinktion, der sozialen Verortung der Schülerinnen und Schüler (Käsler, 2005). Sprachdidaktische Forschung sollte sich deshalb auch mit der Reproduktion von Bildungsungleichheit im Unterricht befassen. Dazu bieten Mikroanalysen von sprachlichen Bildungsprozessen in Kombination mit ethnografischen Untersuchungen ihrer institutionellen Rahmenbedingungen eine vielversprechende Möglichkeit. Entscheidend für den Nutzen solcher Forschungsvorhaben ist letztlich ihre methodische Qualität: die methodologische Klarheit; die Passung von Frage- 
stellung, Daten und Methoden; die Auswahl geeigneter Erhebungs- und Auswertungsverfahren; die Triangulation mehrerer Perspektiven; ein zirkuläres, daten- und theorieverankertes Vorgehen; die Reflexion und transparente Darstellung des Forschungsprozesses. Eine theoretisch und methodisch rigorose verstehend-rekonstruierende Forschung hat unserer Ansicht nach das Potential, der Komplexität der sprachlichen Förderung gerecht zu werden und der aktuellen Tendenz entgegenzuwirken, den Sprachunterricht den Formen der Leistungsmessung anzupassen (Feilke, 2011).

\section{Perspektiven für die Professionalisierung der Lehrpersonen}

Wie lässt sich die Praxis der Sprachförderung in der Schule beeinflussen? Neben den traditionellen Formen der Inputsteuerung (Lehrplänen und Lehrmittel) haben in den letzten Jahren Instrumente zur Output-Messung (Leistungstests und Kompetenzstandards) an Bedeutung gewonnen. Beide Ansätze setzen auf der strukturellen Ebene des Bildungssystems an. Die Wirksamkeit der Sprachförderung wird aber auch und in hohem Masse von der Prozessqualität, d.h. von der Ausgestaltung konkreter Sprachfördersituationen im Unterricht bestimmt (Kuger \& Kluczniok, 2009). Damit rückt die Professionalität der Lehrpersonen in den Vordergrund.

Das berufliche Handeln von Lehrpersonen im Kindergarten ist bislang weitgehend unerforscht. Es ist ungeklärt, wie die in der Ausbildung erworbenen Qualifikationen im beruflichen Alltag umgesetzt werden (Rabe-Kleberg, 2010). Verstehend-rekonstruierende Untersuchungen von Prozessen der Sprachförderung ermöglichen es, subjektive Perspektiven und latente Handlungsmuster zu identifizieren und so die Beziehungen zwischen professionellem Wissen und Handeln der Lehrpersonen aufzuklären. Auf dieser Grundlage könnten zukünftige Aus- und Weiterbildungsangebote genauer auf den Qualifikationsbedarf ausgerichtet und verbindlicher moderiert werden.

Ziel müsste es sein, die Lehrpersonen im Hinblick auf eine «rationale Pädagogik» (Bourdieu \& Passeron, 1971, S. 88) zu qualifizieren, die Bildungsungleichheit eher abbaut, als sie zu reproduzieren. Dazu müssten Lehrpersonen die Zusammenhänge von Sprache und Bildungsungleichheit verstehen und ihre eigene soziale Herkunft und pädagogische Praxis kritisch reflektieren. Es ginge also im Kern darum, sie beim Aufbau einer forschenden Haltung zu unterstützen, die ihnen a) eine kritisch-distanziertes Verhältnis zu ihrer eigenen Praxis, b) einen neugierig fragenden Blick auf ihnen vertraute und unvertraute Milieus und c) eine theorie- und datenverankerte Reflexion ermöglicht. Aus einer solchen Haltung heraus wäre es ihnen leichter möglich, im Wissen um die gesellschaftlichen Rahmenbedingungen und Positionskämpfe auf die unterschiedlichen Bildungserfahrungen der Kinder einzugehen und sie beim Erwerb schulsprachlicher Fähigkeiten adaptiv zu unterstützen. Eine verstehend-rekonstruierende sprachdidaktische Forschung könnte zu einer so verstandenen Professionalisierung von Kindergarten-Lehrpersonen Wesentliches beitragen. 


\section{Anmerkungen}

1 Die Beobachtungssequenzen dienen der Veranschaulichung der im Text diskutierten theoretischen Konzepte und werden in diesem Sinne kommentiert. Eine Darstellung der Analyseverfahren ist an dieser Stelle aus Platzgründen nicht möglich.

2 In der Deutschschweiz ist der Kindergarten inzwischen in vielen Kantonen in das öffentliche Schulsystem integriert.

3 Für eine aktuelle Darstellung und Diskussion linguistischer Ansätze zur Analyse von Unterrichtskommunikation vgl. Becker-Mrotzek \& Vogt (2009).

\section{Literatur}

Amann, K. \& Hirschauer, S. (Hrsg.). (1997). Die Befremdung der eigenen Kultur. Ein Programm. In S. Hirschauer \& K. Amann (Hrsg.). Die Befremdung der eigenen Kultur. Zur ethnographischen Herausforderung soziologischer Empirie (S. 7-52). Frankfurt am Main: Suhrkamp.

Auer, P. (1999). Sprachliche Interaktion. Eine Einführung anhand von 22 Klassikern. Tübingen: Max Niemeyer.

Becker-Mrotzek, M. \& Vogt, R. (2009). Unterrichtskommunikation. Linguistische Analysemethoden und Forschungsergebnisse. Reihe Germanistische Arbeitshefte (38). Tübingen: Niemeyer.

Bernstein, B. (1988). Social class, codes and communication. In U. Ammann, N. Dittmar \& K. Mattheier (Hrsg.), Soziolinguistik II (S. 563-578). Berlin: De Gruyter.

Bourdieu, P. \& Passeron, J.-C. (1971). Die Illusion der Chancengleichheit. Untersuchungen zur Soziologie des Bildungswesens am Beispiel Frankreichs. Stuttgart: Klett.

Bourdieu, P. (2001). Die konservative Schule. Die soziale Chancenungleichheit gegenüber Schule und Kultur. In M. Steinrücke (Hrsg.), Pierre Bourdieu. Wie die Kultur zum Bauern kommt. Über Bildung, Schule und Politik (S. 25-52). Hamburg: VSA.

Bruner, J. (2002). Wie das Kind sprechen lernt (U. Aeschbacher, Übersetzer). Bern: Hans Huber. (Originalarbeit erschienen 1983).

Bude, H. (2006). Stichwort Fallrekonstruktion. In R. Bohnsack, W. Marotzki \& M. Meuser (Hrsg.), Hauptbegriffe Qualitativer Sozialforschung (S. 60-61). Opladen: Leske und Budrich.

Cairney, T. H. \& Ashton, J. (2006). Three families, multiple discourses: Examining differencies in the literacy practices of home and school. Linguistics and Education, 13 (3), 303-345.

Ehlich, K. (2005). Sprachaneignung und deren Feststellung bei Kindern mit und ohne Migrationshintergrund - was man weiss, was man braucht, was man erwarten kann. In K. Ehlich (Hrsg.), Anforderungen an Verfahren der regelmässigen Sprachstandsfeststellung als Grundlage für die frühe und individuelle Förderung von Kindern mit und ohne Migrationshintergrund (S. 11-63). Berlin: Bundesministerium für Bildung und Forschung.

Erard, S. \& Schneuwly, B. (2005). La didactique de l'oral: Savoirs ou compétences? In J.-P. Bronckart, E. Bulea \& M. Pouliot (Éd.), Repenser l'enseignement des langues: Comment identifier et exploiter les compétences? (pp. 69-97). Villeneuve d'Ascq cedex: Presses Universitaires du Septentrion.

Feilke, H. (2002). Die Entwicklung literaler Textfähigkeit. Ein Forschungsbericht. Siegener Arbeitspapiere zur Aneignung sprachlicher Strukturformen SPASS, 10, 1-24.

Feilke, H. (2011). Literalität und literale Kompetenz: Kultur, Handlung, Struktur. leseforum.ch Online-Plattform für Literalität, 1. Zugriff am 5.10.2011 unter http://www.leseforum.ch/myUploadData/files/2011_1_Feilke.pdf

Felder, E. (2003). Sprache als Medium und Gegenstand des Unterrichts. In U. Bredel, H. 
Günther, P. Klotz, J. Ossner \& G. Siebert-Ott (Hrsg.), Didaktik der deutschen Sprache (S. 42-51). Paderborn: Schöningh.

Flick, U. (2002). Qualitative Sozialforschung. Eine Einführung. Reinbek bei Hamburg: Rowohlt Taschenbuch.

Flick, U. (2006). Stichwort Triangulation. In R. Bohnsack, W. Marotzki \& M. Meuser (Hrsg.), Hauptbegriffe Qualitativer Sozialforschung (S. 161-162). Opladen: Leske und Budrich.

Flick, U. (2008). Triangulation. Eine Einführung (2. Aufl.). Wiesbaden: VS.

Fried, L. (2010). Sprachliche Bildung. In M. Stamm \& D. Edelmann (Hrsg.), Frühkindliche Bildung, Betreuung und Erziehung (S. 155-175). Zürich: Rüegger.

Garfinkel, H. (1967). Studies in ethnomethodology. Englewood Cliffs: Prentice Hall.

Gebauer, G. \& Wulf, Ch. (1998). Spiel, Ritual, Geste: Mimetische Grundlagen sozialen Handelns. Reinbek bei Hamburg: Rowohlt.

Geertz, C. (2003). Dichte Beschreibung. Beiträge zum Verstehen kultureller Systeme. Frankfurt am Main: Suhrkamp.

Giraudeau, C. \& Florin, A. (2002). Interactions de tutelle dans différents modes d'accueil préscolaires. In M. Trinquier \& M. Zerbato-Poudou (Éd.), Le préscolaire en question: Questions sur les pratiques (pp. 45-57). Toulouse: Presses universitaires du Mirail (Les dossiers des sciences de l'éducation $\mathrm{N}^{\circ} 7$ ).

Grossenbacher, S. (2007). Vorschulstufe: Vom Stiefkind zum «shooting star» im Bildungswesen? Referat anlässlich der Tagung «Erziehung und Bildung 4 bis 8», Kartause Ittingen, 21.06. 2007.

Gukenbiehl, H. L. (2002). Institution und Organisation. In H. Korte \& B. Schäfers (Hrsg), Einführung in Hauptbegriffe der Soziologie (6., erweiterte Auflage) (S. 143-159). Opladen: Leske und Budrich.

Heath, S. B. (1983). Ways with words. Language, life, and work in communities and classrooms. Cambridge University Press.

Hitzler, R. (2006). Stichwort Ethnografie. In R. Bohnsack, W. Marotzki \& M. Meuser (Hrsg.), Hauptbegriffe Qualitativer Sozialforschung (S. 48-51). Opladen: Leske und Budrich.

Holland, D. \& Cole, M. (1995). Between discourse and schema: Reformulating a cultural-historical approach to culture and mind. Anthropology \& Education Quarterly, 26 (4), 475-490.

Hudelot, C. \& Froment, M. (2006). Soutien langagier dans deux milieux institutionnels, la crèche et l'école maternelle. La lettre de l'AiRDF, 39 (2), 10-14.

Isler, D. \& Künzli, S. (2008). Lernwelten Literacies. Förderung konzeptioneller Literalität im Kindergarten. In B. Hofmann \& R. Valtin (Hrsg.), Checkpoint Literacy. Tagungsband 1 zum 15. Europäischen Lesekongress 2007 in Berlin (S. 77-88). Berlin: Deutsche Gesellschaft für Lesen und Schreiben.

Isler, D. \& Künzli, S. (2010). Schulische Praktiken in der Vorschule. Angebote zum Einüben eines schulischen Habitus in einem Deutschschweizer Kindergarten am Beispiel der Förderung von Sprache und Literalität. In A. Brake \& H. Bremer (Hrsg.), Alltagswelt Schule. Die soziale Herstellung schulischer Wirklichkeiten (S. 211-229). Weinheim: Juventa.

Käsler, D. (2005). Sprachbarrieren im Bildungswesen. In P. Berger \& H. Kahlert (Hrsg.), Institutionalisierte Ungleichheiten. Wie das Bildungswesen Chancen blockiert (S. 130-154). Weinheim: Juventa.

Knoblauch, H. (2008). Videographie und Interaktionsanalyse. Handout zur Methodenwerkstatt «Videoanalyse» an der Pädagogischen Hochschule Zürich 26.-28.06.2008. Zürich.

Koch, P. \& Oesterreicher, W. (1994). Schriftlichkeit und Sprache. In H. Günther \& O. Ludwig (Hrsg.), Schrift und Schriftlichkeit (Band 1) (S. 587-604). Berlin: De Gruyter.

Kowal, S. \& O'Connell, C. (2008). Zur Transkription von Gesprächen. In U. Flick, E. von Kardorff \& I. Steinke (Hrsg.), Qualitative Forschung (S. 437-447). Reinbek bei Hamburg: Rowohlt. 
Künzli, S., Isler, D. \& Leemann, R. (2010). Frühe Literalität als soziale Praxis - Analyse von Mikroprozessen der Reproduktion von Bildungsungleichheit. Zeitschrift für Soziologie der Erziehung und Sozialisation, 30 (1), 60-73.

Kuger, S. \& Kluczniok, K. (2009). Prozessqualität im Kindergarten - Konzept, Umsetzung und Befunde. In H. Rossbach \& H. Blossfeld (Hrsg), Frühpädagogische Förderung in Institutionen (S. 159-178). Wiesbaden: VS.

Lahire, B. (1995). Tableaux de familles. Presses Universitaires de Lyon.

Meuser, M. (2006). Stichwort rekonstruktive Sozialforschung. In R. Bohnsack, W. Marotzki \& M. Meuser (Hrsg.), Hauptbegriffe Qualitativer Sozialforschung (S. 140-142). Opladen: Leske und Budrich.

Moser, U. (2005). Lernbedingungen in Schulklassen zu Beginn der 1. Klasse. In U. Moser, M. Stamm \& J. Hollenweger (Hrsg.), Für die Schule bereit? Lesen, Wortschatz, Mathematik und soziale Kompetenzen beim Schuleintritt (S. 167-185). Oberentfelden: Sauerländer.

Moser, U., Keller, F. \& Zimmermann, P. (2008). Soziale Ungleichheit und Fachleistungen. In U. Moser \& J. Hollenweger (Hrsg.), Drei Jahre danach. Lesen, Wortschatz, Mathematik und soziale Kompetenzen am Ende der dritten Klasse (S. 115-151). Oberentfelden: Sauerländer.

OECD (2006). Starting strong II. Early Childhood Education and Care. Paris: OECD.

OECD. (2010). PISA 2009. Results: Overcoming social background - Equity in learning opportunities and outcomes (Volume II). Retrieved February 01, 2011, from http://dx.doi.org/10.1787/9789264091504-en

Polanyi, M. (1985). Implizites Wissen. Frankfurt am Main: Suhrkamp.

Rabe-Kleberg, U. (2010). Bildungsarmut von Anfang an? Über den Beitrag des Kindergartens im Prozess der Reproduktion sozialer Ungleichheit. In H. Krüger, U. Rabe-Kleberg, R. Kramer \& J. Budde (Hrsg.), Bildungsungleichheit revisited. Bildung und soziale Ungleichheit vom Kindergarten bis zur Hochschule (S. 45-54). Wiesbaden: VS.

Reichertz, J. (2006). Stichwort Abduktion. In R. Bohnsack, W. Marotzki \& M. Meuser (Hrsg.), Hauptbegriffe Qualitativer Sozialforschung (S. 11-14). Opladen: Leske und Budrich.

Rossbach, H., Klugcniok, K. \& Kuger, S. (2008). Auswirkungen eines Kindergartenbesuchs auf den kognitiv-leistungsbezogenen Entwicklungsstand von Kindern. In H.-G. Rossbach \& H.-P. Rossfeld (Hrsg.), Frühpädagogische Förderung in Institutionen. Zeitschrift für Erziehungswissenschaft, 22 (Sonderheft), 139-158.

Strauss, A. \& Corbin, J. (1996). Grounded Theory: Grundlagen Qualitativer Sozialforschung. Weinheim: Beltz (PVU).

Thévenaz-Christen, T. (2005). Les prémices de la forme scolaire. Études d'activités langagières orales à l'école enfantine genevoise. Université de Genève.

Tomasello, M. (1999). The cultural origins of human cognition. Cambridge: Harvard University Press

Wannack, E., Sörensen Criblez, B. \& Gilliéron Giroud P. (2006). Frühe Einschulung in der Schweiz. Ausgangslage und Konsequenzen (Studien + Berichte 26A). Bern: EDK.

Wulf, C. (2006). Stichwort Mimesis. In R. Bohnsack, W. Marotzki \& M. Meuser (Hrsg.), Hauptbegriffe Qualitativer Sozialforschung (S. 117-119). Opladen: Leske und Budrich.

Wygotski, L. (1986). Denken und Sprechen (G. Sewekow, Übersetzer). Frankfurt am Main: Fischer Taschenbuch. (Originalarbeit erschienen 1934).

Schlagworte: Bildungsungleichheit, Sprachliche und literale Praktiken, Familie, Kindergarten, Rekonstruktive Sozialforschung, Sprachdidaktik, Bildungssoziologie 


\section{Enquête sur les pratiques langagières au sein des familles et à l'école enfantine: fondements théoriques et méthodologiques}

\section{Résumé}

En Suisse, on observe dès l'entrée à l'école un rapport étroit entre le milieu social et les performances linguistiques des enfants. Pourtant, on en sait très peu sur leurs expériences d'apprentissage à la maison et dans les structures préscolaires. Cet article traite des fondements théoriques et méthodologiques de l'étude des pratiques langagières. La première partie aborde les prémisses et les implications méthodologiques. Dans la deuxième partie, nous présentons la notion de langage en tant que pratique sociale et illustrons notre propos à l'aide de matériaux issus de notre projet de recherche «Lernwelten-literacies». En conclusion, nous esquissons quelques perspectives pour de futures recherches en éducation linguistique et pour la formation des enseignants.

Mots clés: Equité dans l'éducation, pratiques langagières et littérales, famille, école enfantine, méthodologique reconstructive en sciences sociales, éducation au langage et à la littératie, sociologie de l'éducation

\section{Un'inchiesta sulle pratiche linguistiche nelle famiglie e nella scuola dell'infanzia. Fondamenti teorici e metodologici}

\section{Riassunto}

In Svizzera, già all'inizio della scolarizzazione, si registra una forte correlazione tra la provenienza sociale dei bambini e le loro competenze linguistiche. Tuttavia, si sa poco a proposito delle loro esperienze di apprendimento linguistico in famiglia e nel periodo pre-scolastico. Questo articolo tratta dei fondamenti metodologici e teorici per investigare tali pratiche. Nella prima parte vengono discusse delle premesse metodologiche e le loro implicazioni. Nella seconda parte presentiamo il concetto di lingua come pratica sociale e la illustriamo tramite una selezione di dati del progetto Lernwelten - literacies (Mondi di significato alfabetizzazione). Infine, vengono delineate alcune prospettive per la ricerca sull'insegnamento delle lingue e sulla professionalizzazione dei docenti.

Parole chiave: Equità educativa, pratiche linguistiche e letterarie, scuola dell'infanzia, metodologie ricostruttive nelle scienze sociali, educazione al linguaggio e all'alfabetizzazione, sociologia dell'educazione. 


\section{Investigating language pratices in families and kindergarten classrooms: Theoretical and methodological foundations}

\section{Summary}

In Switzerland, there is a strong correlation between the social backgrounds and language outcomes of children already at school entry. At the same time, very little is known about their home learning experiences and language education in preschool institutions. This article deals with methodological and theoretical foundations pertinent to investigate language practices. In the first section, methodological premises and implications are discussed. In the second section, we present the notion of laguage as social practice and illustrate our understanding by means of selected data from our research project "Worlds of learning - literacies". Finally, some perspectives for future classroom research on language education and for teacher qualification are outlined.

Key words: Equity in education, language and literacy practices, family, kindergarten, reconstructive methodology in the social sciences, language and literacy education, educational sociology 